\title{
The Supernebula and Protoglobular Cluster in NGC 5253
}

\author{
Jean L. Turner \\ UCLA, Division of Astronomy \& Astrophysics, Los Angeles CA 90095
}

\begin{abstract}
A compact and bright radio and infrared source within the starburst center of the dwarf galaxy NGC 5253 appears to be an extremely large compact HII region. The high density $\left(n_{e} \sim\right.$ few $\left.\times 10^{4} \mathrm{~cm}^{-3}\right)$ and size $(\sim 1-2 \mathrm{pc})$ of the nebula require the ionization equivalent of at least 4000 O7 stars. We suggest that this optically obscured nebula is a forming super star cluster, or protoglobular cluster, with an age of a few hundred thousand years.
\end{abstract}

\section{The Dwarf Galaxy NGC 5253 and its Luminous Starburst}

NGC 5253 is a dwarf I0/S0 galaxy, located near the large spiral galaxy M83, at a distance of $\sim 4 \mathrm{Mpc}$. It is well known for the anomalous dust lane extending into the central part of the galaxy (e.g., van den Bergh 1980, Graham 1981). NGC 5253 is presently undergoing a starburst of infrared luminosity $L_{I R} \sim$ $2 \times 10^{9} L_{\odot}$ (Beck et al. 1996). The starburst consists of an extended region of young super star clusters (Gorjian 1996; Calzetti et al. 1997) and bright, filamentary $\mathrm{H} \alpha$ emission (Bohuski et al. 1972; Calzetti et al. 1997). Surrounding the galaxy are numerous intermediate age clusters (Caldwell \& Phillips 1989), indicating that the dwarf galaxy has had a complex history.

In the center of the starburst, there is an optically obscured, radio bright HII region. This HII region has no obvious optical counterpart. The nebula was first discovered in arcsecond resolution VLA images (Beck et al. 1996; Turner et al. 1998). The radio flux indicated a Lyman continuum rate requiring thousands of OB stars within the $10 \mathrm{pc}$ beam. Subsequent, higher resolution VLA images revealed that this source is even more compact: $2 \mathrm{pc} \times 1 \mathrm{pc}$ in extent (Turner et al. 2000.) The electron density is remarkably high for a nebula of this size: $n_{e} \sim 4 \times 10^{4} \mathrm{~cm}^{-3}$, a density characteristic of the youngest "compact HII regions" in our Galaxy, regions that are typically a few hundred thousand years old (Wood \& Churchwell 1989). However, in spite of the abundant circumstantial evidence in favor of an HII region interpretation, other sources of radio emission for this unusual source, albeit unlikely, were possible (Turner et al. 1998, 2000).

Using the Keck LWS in imaging mode, we have confirmed that the radio source is an HII region (Gorjian et al. 2000). Diffraction limited imaging at 12 and $18 \mu \mathrm{m}$ shows a bright infrared source, with flux densities of 2.1 and $10.4 \mathrm{Jy}$, respectively, and size $<0.5^{\prime \prime}$. The ratio of mid-IR to radio flux, $S_{\text {IR }} / S_{2 \mathrm{~cm}} \sim 100$ is typical of those observed in Galactic HII regions. The size of the IR nebula is consistent with the radio size. The source is clearly not a supernova remnant, and extremely unlikely to be an AGN. The overwhelming odds are that it is an 
HII region. It is probably the source of the strong Brackett lines (Kawara et al. 1989) and radio recombination lines (K. Anantharamaiah, private commun.) in NGC 5253, although the lower resolutions of these data do not allow a direct spatial comparison with the radio and mid-IR source.

\section{The Supernebula in NGC $\mathbf{5 2 5 3}$}

This nebula provides a remarkable demonstration of how compact starburst emission can be. The radio and IR observations require a Lyman continuum flux of $4 \times 10^{52} \mathrm{~s}^{-1}$, the equivalent of 4000 O7 stars. If the IMF is Salpeter, as seems to hold for R136 (Massey \& Hunter 1998; H. Zinnecker, private commun.), then there are over a million stars localized to this $1-2 \mathrm{pc}$ region. If the stars are more distributed, then the excitation requirements on the cluster are even more extreme. This tiny $1 \times 2$ pc nebula is responsible for $80 \%$ of the total midinfrared IRAS flux of NGC 5253, and at least $30 \%$ of the total infrared luminosity of the entire galaxy. Unless there is some unusual source of confinement (there is, surprisingly, no evidence of any molecular gas in the vicinity of the HII region: Turner, Beck, \& Hurt 1997), then the nebula, like Galactic compact HII regions, is at most a few hundred thousand years old. It is therefore likely that in this source we are witnessing the birth of a super star cluster, and a potential protoglobular cluster. Similar nebulae, although not as large as the one in NGC 5253, have been seen in He 2-10 (Kobulnicky \& Johnson 1999) and NGC 2146 (Tarchi et al. 2000).

\section{References}

Beck, S. C., Turner, J. L., Ho, P. T. P., Lacy, J. H., \& Kelly, D. 1996, ApJ, 457, 610

Bohuski, T. J., Burbidge, E. M., Burbidge, G. R., \& Smith, M. G. 1972, ApJ, 175,329

Caldwell, N., \& Phillips, M. M. 1989, ApJ, 338, 789

Calzetti, D., Meurer, G. R., Bohlin, R. C., Garnett, D. R., Kinney, A. L., Leitherer, C., \& Storchi-Bergmann, T. 1997, AJ, 114, 1834

Gorjian, V. 1996, AJ, 112, 1886

Gorjian, V., Turner, J. L., \& Beck, S. C. 2000, submitted

Graham, J. A. 1981, PASP, 93, 552

Kawara, K., Nishida, M., \& Phillips, M. M. 1989, ApJ, 337, 230

Kobulnicky, H. A., \& Johnson, K. E. 1999, AJ, 527, 154

Massey, P. \& Hunter, D. A. 1998, ApJ, 493, 180

Tarchi, A. et al. 2000, A\&A, 358, 95

Turner, J. L., Beck, S. C., \& Ho, P. T. P. 2000, ApJ, 532, L109

Turner, J. L., Beck, S. C., \& Hurt, R. L. 1997, ApJ, 474, L11

Turner, J. L., Ho, P. T. P., \& Beck, S. C. 1998, AJ, 116, 1212

van den Bergh, S. 1980, PASP, 92, 122

Wood, D. O. S., \& Churchwell, E. 1989, ApJS, 69, 831 